\title{
How to Simulate Galactic Outflows?
}

\author{
Paramita Barai \\ INAF - Osservatorio Astronomico di Trieste, Via G. B. Tiepolo 11, I-34143 Trieste, Italy \\ email: pbarai@oats.inaf.it
}

\begin{abstract}
A challenge in cosmological simulations is to formulate a physical model of starformation (SF) and supernovae (SN) feedback which produces galactic outflows like that widely observed. In several models an outflow velocity $\left(v_{\text {out }}\right)$ and mass loading factor $(\eta)$ are input to the sub-resolution recipe. We present results from our MUPPI model, which uses local properties of gas, and is able to develop galactic outflows whose properties correlate with global galaxy properties, consistent with observations; demonstrating a significant improvement in such work.
\end{abstract}

Keywords. Cosmology: theory - Methods: Numerical - Galaxies: formation

\section{Introduction}

We explore our novel sub-resolution model for SF and SN feedback, MUPPI [MUltiPhase Particle Integrator] (Murante et al. 2010, 2014), embedded in the GADGET3 code, which uses only local properties of the gas. Unlike popular adaptation in the literature, our model has no input expression of $v_{\text {out }}$ and $\eta$ for SN feedback.

\section{Conclusions}

We measure properties of SN-driven galactic outflows over redshifts $z=1-5$ in cosmological hydrodynamical simulations (Barai et al. 2013, 2014). The MUPPI model generates $v_{\text {out }}$ and mass outflow rate $\left(\dot{M}_{\text {out }}\right)$ exhibiting positive correlations with galaxy mass and with the star formation rate (SFR) (Fig. 1). However, most of the relations present a large scatter. The mass-loading is between $0.2-10$, with an average $\eta \sim 1$.

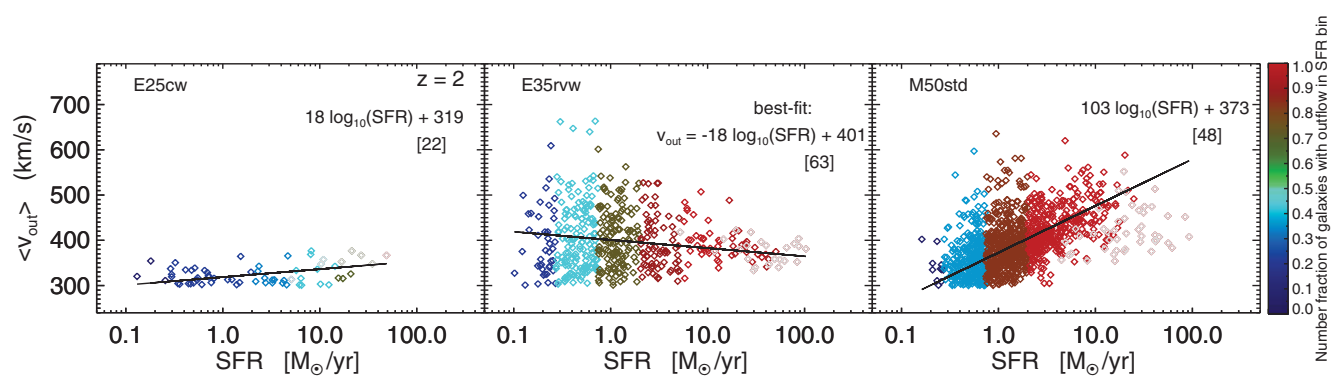

Figure 1. Outflow velocity as a function of SFR of galaxies in three cosmological simulation runs at $z=2$. The black line is the best-fit relation between $v_{\text {out }}$ and $\log _{10}(\mathrm{SFR})$.

\section{Acknowledgements}

I acknowledge support from the ERC Starting Grant "cosmoIGM". 


\section{References}

Barai P. et al., 2013, MNRAS, 430, 3213

Barai P., Monaco P., Murante G., Ragagnin A., \& Viel M., 2014, submitted to MNRAS

Murante G., Monaco P., Giovalli M., Borgani S., \& Diaferio A., 2010, MNRAS, 405, 1491

Murante G., Monaco P., Borgani S., Tornatore L., Dolag K., \& Goz D., 2014, submitted to MNRAS 